\title{
Power Consumption Model for the DSP OAK Processor
}

\author{
Guitton-Ouhamou Patricia, Belleudy Cécile, Auguin Michel \\ Laboratoire d'Informatique, Signaux et Systèmes de Sophia-Antipolis, \\ Les Algorithmes batiment. Euclide, 2000, route des Lucioles-BP 121, \\ 06903 Sophia-Antipolis Cedex, \\ belleudy@i3s.unice.fr, guitton@i3s.unice.fr,auguin@i3s.unice.fr
}

\begin{abstract}
The remarkable growth of personal computing devices, like portable desktops, audio and video products, and wireless communication equipments require high speed processor with low power consumption. In order to reduce power consumption, it is necessary to quantify first this value for current processors. Methods working at logic level require numerous computations to evaluate consumptions of each processor's instruction [6]. For complete and complex applications, these methods are not really applicable. The novelty of our approach is to estimate the power consumption in the same time as the designer describes his application. The objective would be to define a power consumption estimator from the $\mathrm{C}$ language description of the application and a library of processors. In this sense, we propose, as first work, to define a power consumption estimation model at assembly level. Our estimation technique is based on an operator level model of the data path and is compared with measurements on a test board. In this paper, this technique is applied to the OAK DSP ${ }^{\mathrm{TM}}$ processor. To conclude, we propose some writing rules of the assembly code to optimise the power consumption.
\end{abstract}

Key words : DSP processor, power consumption model.

\section{INTRODUCTION}

Previous IC designs constraints were area, performance and cost. Due to the growth of portable devices requiring greater autonomy, power becomes an important factor. In order to get power savings, it is necessary to derive first a power consumption model. Working at gate level requires many hours of simulation for small designs. Applications are generally written in $\mathrm{C}$ language, thus an estimator working at this level allows rapid evaluation in the

The original version of this chapter was revised: The copyright line was incorrect. This has been corrected. The Erratum to this chapter is available at DOI: 10.1007/978-0-387-35597-9_40 
early design steps. Since debugging tools including a C-cross compiler are common for DSP processors, a first approach could be to extract a model at the assembly level. Unfortunately C compilers for DSPs are ineffective [1] due to specific architectures of DSP processors. Our approach is based on a two level estimator:

- The $\mathrm{C}$ program may be compiled in an intermediate representation based on the Register Transfer Language (RTL) [8]. This representation gives the list of operations and their precedences to be executed. The advantage of the RTL representation is that it is independent of the target processor. Unfortunately, this representation doesn't take into account parallel instructions and addressing modes of DSPs. So we consider the VESTIM tool [1]fig1., developed with the support of Phillips semiconductor Sophia. This tool uses the modified RTL representation by grouping operations forming parallel instructions, including addressing modes. The goal of this tool is to estimate the execution time of an application from a C description.

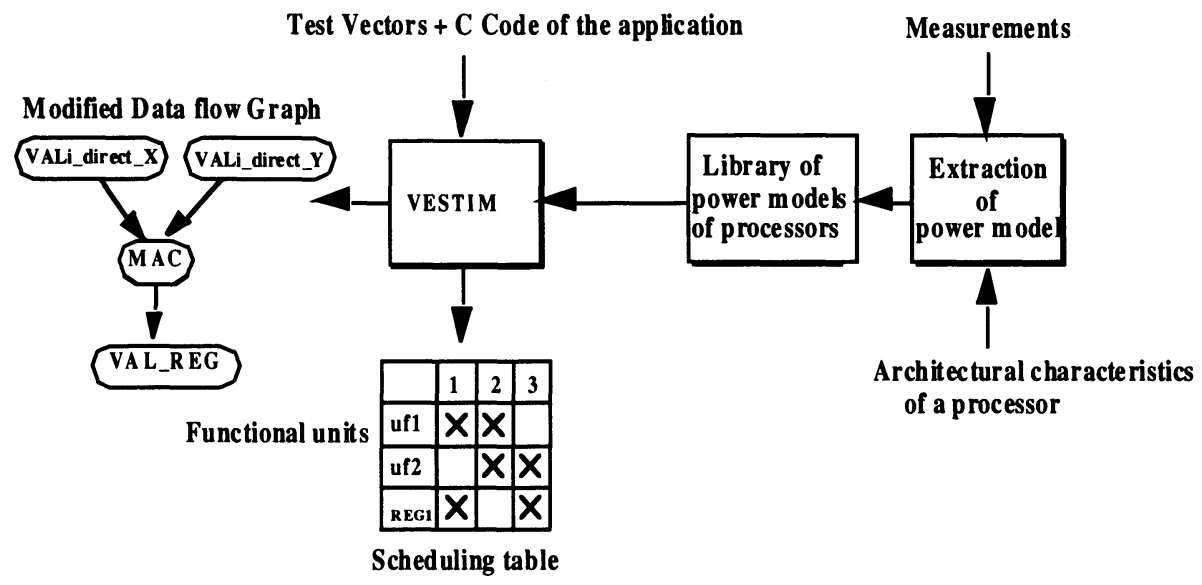

Figure 1. VESTIM tool

- From individual evaluation of power consumption of these operations and the associated addressing modes, the total power for the application can be estimated. In order to know the consumption of one operation, we consider measurements at the assembly level [11][6]. This approach is easy to implement and a power consumption model of the DSP can be deduced. Furthermore, this model could be embedded in current debugging tools, providing a power consumption profiling of the application.

In order to extract this model, we took into account micro-architectural details of the processor. Since the power cost is an increased problem in mobile designs, we choose the OAK DSP core that is embedded in numerous 
designs. Furthermore, this approach (at assembly level) was considered for processors, like SPARClite, MB86934, intel i80486DX, ARM7TDMI [6][11]. As detailed in the sequel, models for RISC cannot be applied for DSPs which are data dependent.

The paper is organised as follows. First, we give the architecture characteristics of the OAK DSP processor and we describe the power measurement method. We then present preliminary results and the consumption model. The consumption due to the base cost of one instruction and the inter-instruction cost due to data path changes between two consecutive instructions. Results for test applications (FFT, FIR) are given to evaluate the accuracy of the estimator. We illustrate that writing rules of the assembly code permit a significant reduction of the power consumption. We conclude with future work.

\section{THE OAK DSP PROCESSOR AND ITS POWER MEASUREMENTS}

The OAK DSP processor [12] is a CMOS 16 bits fixed point DSP processor (figure 2).

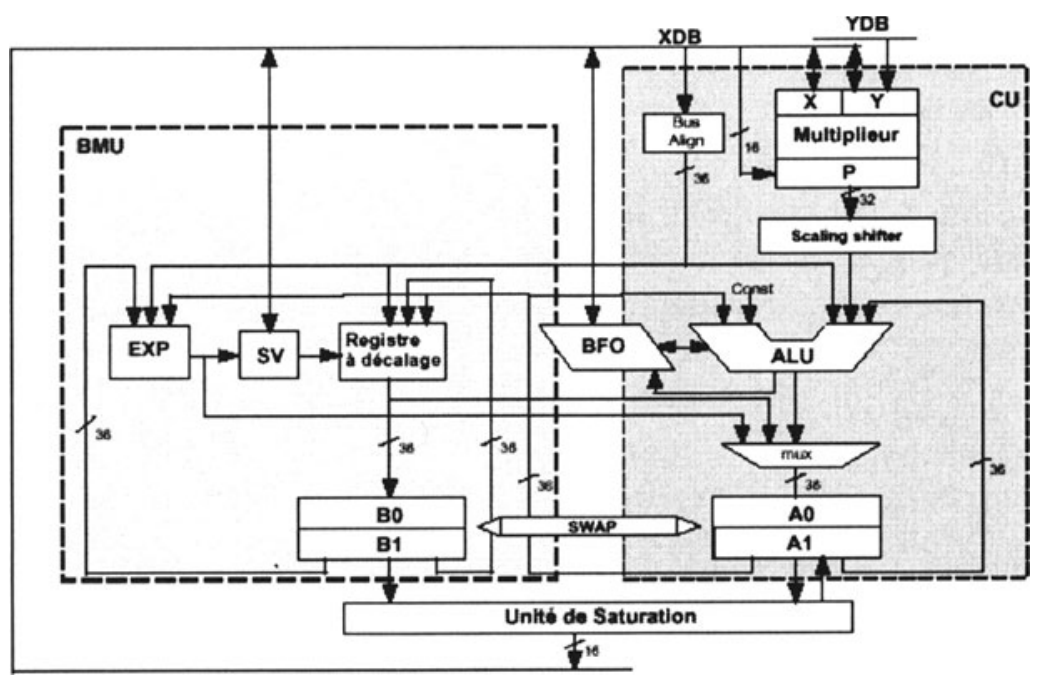

Figure 2. The data path of the $O A K$ DSP core.

It consists briefly of the MPU, the ALU, the barrel shifter, the exponent logic unit, the data address arithmetic unit, four 36-bit accumulators, six data/address registers $\mathrm{r} 0 . . . \mathrm{r} 5$ (16 bits), and two internal memories $\mathrm{X}$ and $\mathrm{Y}$. Busses connecting the registers ri are preloaded to 1 . Measurements are done 
at $40 \mathrm{Mhz}$ with a supply voltage of $3.3 \mathrm{~V}$ for the board and $2.5 \mathrm{~V}$ for the processor core.

In order to evaluate the power consumption, we use the board-based measurement method developed in [11][6]. The average power is given by $\mathrm{P}=\mathrm{I}^{*} \mathrm{Vdd}$ where $\mathrm{I}$ is the average current and Vdd the supply voltage. The energy $E$ is given by: $E=P * T$ where $T$ is the execution time of the program. The basic idea is to measure the consumed current by inserting an ammeter between the power supply and the CPU (the power supply connection to the CPU must be isolated from the reminder of the system).

Evaluation of the energy consumption of an assembly program, requires to differentiate two types of consumptions:

- the base cost of an instruction provided by the consumption of the execution of one instruction,

Measurement principles: for each instruction, we create a test program that contains initialisation of the system and a loop including same instruction repeated 200 times. This number has been determined so that the loop instructions does not modify the measured current value.

- the inter-instruction (or overhead) cost due to the changes in the data path activated by two successive instructions.

Measurement principles: The test program contains 200 pairs of instructions.

In a first approach, these measurements must be realised for each instruction, each value and each addressing mode of the operands. Since this work is too exhaustive, classes of instructions are formed. Two instructions in a class activate the same data path in the DSP. For example, operations executed by the ALU and using the same operand addressing belong to the same class.

The evaluation of the power consumption of a program is calculated by [6][11]: $\mathrm{E}=\Sigma_{\mathrm{i}}\left(\mathrm{R}_{\mathrm{i}} * \mathrm{~N}_{\mathrm{i}}\right)+\Sigma_{\mathrm{i}, \mathrm{j}}\left(\mathrm{O}_{(\mathrm{i}, \mathrm{j})} * \mathrm{~N}_{(\mathrm{i}, \mathrm{j})}\right)+\Sigma_{\mathrm{k}} \mathrm{E}_{\mathrm{k}}$

Where: $\mathrm{O}_{(\mathrm{i}, \mathrm{j})}$ : is the overhead cost due to execution of instruction $\mathrm{i}$ followed by instruction $\mathrm{j}$;

$\mathrm{Ri}$ : the base cost of instruction $\mathrm{i}$;

$\mathrm{Ni}$ : number of cycles to execute instruction $\mathrm{i}$.

Ek represents the consumption due to lost cycles, for example, cache and pipeline stalls. 


\section{BASE COST OF A SINGLE INSTRUCTION}

In [6][11], the authors approximate the base power consumption of the DSP Fujitsu $3.3 \mathrm{~V}, 0.5 \mathrm{~m}, 40 \mathrm{MHz}$ by an average value for each class of instructions. The range of variation inside a class is small, less than $10 \%$.

For the OAK DSP processor, the current values inside a class can vary up to $300 \%$. Its approximation by an average constant value would lead to make an error of $100 \%$. It is necessary to develop a more accurate model that takes into account the architectural characteristics of the processor. This requires to identify the power consumption sources.

\subsection{The consumption sources of the DSP}

Considering the architectural characteristics, two sources of variation were identified:

a) the preload of the busses: the bits equal to 0 consume more than those equal to 1.

This preload allows a faster clock rate. Indeed, in CMOS technology forcing busses to the logical level 0 is faster than forcing them to the logical level 1 .

The preload of the busses is activated only when operands use the data/address registers of the DSP.

To evaluate the effect of the preload of busses, the current values of an instruction are measured first using accumulator input operands and next using data/address register input operands. The difference between these two values represents the consumption due to the preload bus activity. We notice that in the worst case, the current can double when data/address register operands are used. The preload of busses is an important factor of consumption for this processor.

In this study, we consider different numbers and positions of bits equals to 0 in the input operands.

b) The switching activity:

Another part of the consumption is due to the transitions of the bits, varying at each cycle from 0 to 1 and conversely.

This switching activity results from the charges and discharges of the capacities of transistors, that all put together corresponds to the switching capacity $\mathrm{C}$ of the circuit, giving a power consumption proportional to: $\mathrm{CV}^{2}$ where $V_{d d}$ is the supply voltage.

Measurements lead to conclude that the consumed power of the DSP depends mainly on the switching activity of the signals. Let $\alpha$ be the average 
number of transitions per cycle. The power $\mathrm{P}_{\text {unit }}$ consumed by a unit of the processor with a clock $f_{\text {clk }}$ is [3][10]: $P_{\text {unit }}=\alpha * f_{\text {clk }} *\left(C * V_{d d}{ }^{2}\right)$.

The consumption of energy generated by the execution of an application for all the units of the processor [3], [10] is:

Pswitching $=\Sigma_{0 \text { to n }} \alpha_{\mathrm{l}} * \mathrm{f}_{\mathrm{clk}} *\left(\mathrm{C}_{\mathrm{i}} * \mathrm{~V}_{\mathrm{dd}}{ }^{2}\right)$.

with:

- $\mathrm{n}$ : number of units of the processor;

- $\alpha_{1}$ : average number of bit transitions per cycle of the ith unit;

- Ci: output capacity of the considered unit.

However the output capacity of each unit is unknown. By measuring the current consumed by an instruction, we can estimate the power induced by the switching activity by changing the number of switching bits of the input operands. The influence of the positions of the switching bits was also tested.

In the worst case, we notice that the current may be multiplied by 1.5 when all bits switch compared to the current with no bit changes (to avoid effect of the preload of busses, operands are accumulators). In order to take into account the preloaded busses and switching activity, consumption sources, the following instruction current model is:

$\mathrm{I}=\mathrm{I}_{\text {cst }}+\mathrm{I}_{\text {preloaded bus }}+\mathrm{I}_{\text {switching activity }}(1)$ where $\mathrm{I}_{\text {cst }}$ is the current measured with no switching activity.

In the next section, we give the models for common instructions. For more details refer to [4].

\subsection{Consumption model for the instructions relative to the ALU}

We refine the previous formula (1). The measurements of the current due to the preloading of busses show that all the bits have the same influence on the consumption with the exception of the sign bit. The model for the preloaded bus is:

$$
I_{\text {preloaded bus }}=N^{*} I_{\text {bus unit }}+I_{\text {sign extension }}
$$

where: $\mathrm{N}$ is the number of bits equal to 0 in the input data.

I $_{\text {bus unit: }}$ elementary current (by bit) due the preload of the busses.

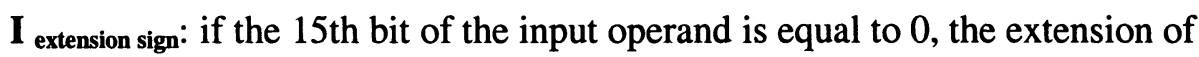
the sign entails the following 20 bits to 0 . If the 15 th bit is equal to $1, I_{\text {sign extension }}=0$. 


\section{These basic currents are measured as follows:}

- $I_{\text {bus unit }}=\left(I_{\text {and } r 1=0, a 0}-I_{\text {and } r 1=7 F F F, a 0}\right) / 15$, second operand is equal to 0 so that there is no switching activity.

- $\mathrm{I}_{\text {extension sign: }}$ If the $15^{\text {th }}$ bit is equal to $0, \mathrm{E}_{\mathrm{xtension} \mathrm{sign}}=\left(I_{\text {and } r l=7 F F F, a 0}-I_{\text {and } r l}=\right.$ FFF7,a0) Else $\mathrm{I}_{\text {extension sign }}=0$.

- $\mathbf{I}_{\text {switching activity }}=\left(I_{\mathrm{xor} a \mathrm{l}=1, \mathrm{a} 0}-I_{\mathrm{xor} \mathrm{al}=0, \mathrm{a} 0}\right)$.

This model is applicable for arithmetic and logic instructions executed by the ALU such as add, sub, cmp, and, or, xor.... (instructions of this class are labelled $A L U$ ). It concerns the switching activity of outputs of the functional units.

\section{Accuracy of the model:}

For these instructions, the estimated values of the consumption are compared with real values:

- for logic instructions (and, or, xor), the worst case reveals an error of $2.5 \%$.

- for arithmetic instructions, the error is $6 \%$ in the worst case.

\subsection{Consumption model for the instructions invoking the multiplier (MPU)}

We consider the basic model: $\mathbf{I}=\mathbf{I}_{\mathrm{cst}}+\mathbf{I}_{\text {preloaded bus }}+\mathbf{I}_{\text {switching activity }}$.

a) Preload of busses: the busses are preloaded when the second operand is a data/address register. As performed for the ALU unit, we changed in the operand data the number of zeros and their position while avoiding switching activity in the multiplier by setting to zero the second operand. In this case, we observe that the number of zeros has a different impact on the power consumption according to the considered slice of 4 bits.

Table 1.

\begin{tabular}{|c|c|}
\hline Number of bits to 0 & $\mathrm{I}_{\text {preloaded bus }(\mathrm{mA})}$ \\
\hline \hline Slice 0 to 3 & $\mathrm{I} 1$ \\
\hline Slice 4 to 7 & $\mathrm{I} 2(<\mathrm{I} 1)$ \\
\hline Slice 8 to 11 & $\mathrm{I} 3(<\mathrm{I} 2)$ \\
\hline Slice 12 to 15 & $\mathrm{I} 4(<\mathrm{I} 3)$ \\
\hline
\end{tabular}

b) The switching activity: the MPU in the OAK is a Booth multiplier, thus only the second operand contributes to the consumed current. 
To estimate the switching activity, we consider a pair of instructions that changes the result on the output bits of the functional unit at each cycle. This measured value is subtracted from the average value of the base costs of each instruction.

Example: $m p y$ y $=1, r 1=0$ and mpy $y=1, r 2=000 F$

At each cycle, four bits change, giving an elementary weight per bit. The method is the following:

$\mathrm{I}_{\mathrm{mpy}, \mathrm{r} 1=0-\mathrm{mpy}, \mathrm{r} 2=\mathrm{F}}=\mathrm{I}_{\mathrm{cst}}+\mathrm{I}_{\text {preloaded bus }}+\mathrm{I}_{\text {switching activity for the } 4 \mathrm{LSB} \text { bits }}$

$I_{\text {mpy,r1 }=0 \text {-mpy,r2=0 }}=I_{\text {cst }}+I_{\text {preloaded bus }}$

The difference between these two currents provides the switching activity for the four LSB bits. The switching activity for the other groups of 4 bits can be measured with the same approach.

We notice that a transition on the signed bit consumed 5 times the consumption due to each other bits.

This model is applicable for instructions executed by the MPU such as mpy, sqr, mac (instructions of this class are labelled $M P U$ )... Notice that the mac instruction has the same model as the instruction mpy and does not result from the addition of the currents consumed by the ALU and the MPU. So parallel instructions lead to reduce the consumption.

Accuracy of the model: Compared to actual measurements, the maximum error is $8 \%$.

\subsection{The mov class}

Let us apply the same formula 1 .

Preload of busses: the preload of busses works as for the ALU. The position of the zeros in the operand has no effect on the consumed current.

The switching activity: the switching activity behaves as for the MPU with a different weight on the sign bit. We notice also that memory writings consume more than memory readings.

Accuracy of the model: the maximum error is $2.8 \%$.

\subsection{Impact of internal memory accesses}

The consumed current analysed in the previous sections is the current consumed by the processor core. The consumption due to memory accesses are measured with an ammeter placed between the memory and the supply voltage. In the executed instructions, some memory accesses are realised. 
Let Imem be the base current of the memory when no accesses are performed by the core, for example, with a nop instruction. The currents generated by memory accesses are:

- memory accesses in XRAM, lead to a current 6\% higher than Imem.

- memory accesses in YRAM, lead to a current $14.4 \%$ higher than Imem.

The difference of consumption between the XRAM and the YRAM memories is due to the interconnection in the data path of the processor. This results are obtained with a size of 8 Kbytes for the $\mathrm{X}$ and $\mathrm{Y}$ memory banks. They illustrate that internal memory accesses do not have a great influence on the total consumption. The model developed in this section is the base cost per instruction, we focus now on the inter-instruction cost.

\section{THE INTER-INSTRUCTION COST}

\subsection{Power model of the inter-instruction cost}

The inter-instruction cost (overhead) is derived from the difference between the current measured on the execution of a couple of different instructions and the sum of the currents associated with each instruction:

$$
\mathrm{I}_{\mathrm{ov}}=\mathrm{I}_{\text {instr1,instr2 }}-\left(\mathrm{I}_{\text {instr1 }}+\mathrm{I}_{\text {instr } 2}\right) / 2 \text {. }
$$

We notice that $\mathrm{I}_{\mathrm{ov}}$ has a range of variation from 1 to 42 .

- For pairs of instructions belonging to the same class, $\mathrm{I}_{\mathrm{ov}}$ is small, excepted for some addressing modes of the mov instruction.

- For pairs of instructions belonging to different classes, $\mathrm{I}_{\mathrm{ov}}$ is significant. To take into account this behaviour we introduce two factors in the model:

- a base current depend on the pair of the consecutive instructions: $\mathbf{I}_{\mathbf{c s t o v}}$,

- a cost dependent on the data value where the bits equal to 1 consume more than the 0 s.

The inter-instruction cost is: $\mathbf{I}_{\text {ov }}=\mathbf{I}_{\text {cstov }}+\mathbf{M}^{*} \mathbf{I}_{\text {unit ov }}$ (2).

where: $\mathrm{M}$ is the number of bits equal to 1 in the operand data.

$I_{\text {unit ov }}$ : elementary current (by bit) consumed by the 1 logic in the data.

The current of $\mathbf{M}^{*} \mathrm{I}_{\text {bus unit ov }}$ is relatively small compared with the bus preload value in the base cost of instructions. If this expression is 
approximated by a constant, the maximum error is $15 \%$. So, to simplify the model, the formula (2) becomes: $\mathbf{I}_{\mathbf{o v}}=\mathbf{I}_{\text {cstov }}+\mathbf{I}_{\text {cst preloaded bus. }}$.

On the contrary to the base cost of instructions, $\mathbf{I}_{\text {cstov }}$ has a great variation according to the considered pair of instructions and the addressing modes. The value $\mathbf{I}_{\text {sst preloaded bus }}$ is constant for all pairs of instructions.

Accuracy of the model: with this model, the maximum error is $16 \%$.

Using this complete instruction level model, we can derive some writing rules to reduce the consumed current.

\section{OPTIMISATION THROUGH WRITING RULES}

\subsection{Optimisation of the base cost of instructions}

Addressing modes have a significant impact on the current consumed by an instruction. Therefore, it is preferable to use the following resources:

- For the ALU : the source operands and addressing modes that lead to reduce the power consumption are: accumulators and registers $(\mathrm{a} 0, \mathrm{a} 1, \mathrm{p})$, short direct, and indirect addressing modes.

- For the multiplication unit : even if the model is not obvious, we can deduce the following writing rule: it is better to use the indirect addressing mode for op2 and/or for op1. As it is the number of 1 in the second operand that determines the consumption, more power swings are observed if the value with maximum of $1 \mathrm{~s}$ is considered for this operand.

- For mov instructions : the current is reduced if the operands involved in the mov instructions are the accumulators a0, a1, and the register $\mathrm{p}$.

\subsection{Optimisation including the overhead}

In a general approach, higher power savings are obtained for successions of instructions belonging to the same class than instructions belonging to different classes. However, these savings are achieved if successive instructions have the same addressing mode. Particularly:

- some pairs of instructions have a low power consumption: mov-mpy, mov-add.

- In contrast, it would be preferable to avoid: mpy-add. 


\section{RESULTS}

On two basic DSP functions (FIR and FFT issued from the DSP library of the OAK processor), we have estimated the consumed current, by adding the base costs of instructions and the associated overheads. This value is then compared with the measurement of the real current on the test board. We obtain:

- the FIR example, the error is $5.6 \%$,

- the FFT radix 2 example, the error is $8.8 \%$.

These results show the power consumption behaviour of the OAK DSP core is well captured in our estimation model and accurate energy consumption can be evaluated for complete program executions. By applying the writing rules described in the previous section, let us try to reduce the consumption of these two DSP functions. The order of assembly instructions is modified in order to group the instructions belonging to the same class. On the two previous examples, the FIR filter and the FFT, a power saving of $14 \%$ and $12.5 \%$ is achieved respectively with the same executing times.

\section{CONCLUSION}

The power estimation model developed in this paper fits well the power behaviour of the OAK DSP core. As introduced in [6], the model is based on a base cost of instructions and on an overhead. Major characteristics of the DSP require to extend the model in [6]: busses of the DSP are preloaded to 1 and for some instructions, the sign bit has a great impact on the switching activity. The model is accurate and gives a maximum error of $8.8 \%$. Although the power consumption is basically evaluated at the data path level it is really an instruction level power consumption estimator. Thus, it can be integrated easily in a assembler/debugger tool allowing the design to get power consumption estimates for its application and for the code section of the application that have the highest contributions to energy.

However, integration of this model in a performance estimator like VESTIM is more challenging since real values of data are not considered in the performance extraction process of VESTIM. One solution could consist in using the Dual Bit Type model [5] to evaluate statistically the ratio of zero bits in the data in order to estimate the switching activity. To extract the 
characteristics needed in the DBT, it is necessary to study statistically some test vectors. We project also to insert this model in a codesign tool (CODEF [2]) to explore hardware/software designs to optimise silicon area, power consumption and performances.

\section{BIBLIOGRAPHY}

[1] Thesis, A. Pegatoquet, "Méthodes d'estimation de performance logicielle: application au développement rapide de code optimisé pour une classe de processeur DSP", Octobre 1999

[2] L. Bianco, M. Auguin, G. Gresset, A. Pegatoquet, "A system prototyping tool for efficient system architecture exploration ", ICSPAT 99, Orlando, novembre 1999.

[3] Chingwei Yeh, Min-Cheng Chang, Shih-Chieh Chang, Wen-Bone Jone,"Gate-level Design exploiting Dual supply voltages for power-driven applications", pp68-71 DAC 1999, New Orleans, LA, USA

[4] Rapport de DEA de patricia Guitton-Ouhamou,"Modélisation de la consommation d'un processeur type DSP", université de Nice-Sophia Antipolis, juin 2000.

[5]Paul E.Landman and Jean M. Rabaey, "Architecture power analysis: The Dual Bit Type Method" , VLSI Systems, vol.3 n.2, June 1995.

[6] M. Tien-Chien Lee, V. Tiwari, S. Malik and M. Fujita, "Power analysis and minimization techniques for embedded DSP software", IEEE Transactions on VLSI Systems, Mars 1997.

[7] Massoud PedramT, "High level Power modeling, estimation and optimization", Tutorial of DAC 1997.

[8]A. Pegatoquet, M. Auguin, L. Bianco, E. gresset, "Rapid Development of optimized DSP Code from a High Level Description through Software Estimations", 36th Design Automation Conference, June 19-24, New Orleans, Louisiana, USA, 1999.

[9] J. Rabaey and Pedram, "Low Power Design Methodologies", Kluwer Academic Polishers, Boston, MA, 1996.

[10] Jan M. Rabaey, "System-level power estimation and optimization-challenges and perspectives", International Symposium on Low-Power Design, pp. 158-160.

[11] V. Tiwari, S. Malik and A. Wolfe, «Instruction level optimisation software», Journal of VLSI Signal Processing Systems, Avril 1996, p.139 à 154.

[12] VVF3500 DSP Core User Manual, VLSI technology, Inc.

[13] C.Brandolese, W.Fornaciari, F.Salice, D.Sciuto, An instruction-level Functionalitybased Energy Estimation Model for 32-bits Microprocessors. 37th Design Automation Conference June 5 - 9, 2000 Los Angeles, CA, proceedings p. 346-351.

\section{ACKNOWLEDGEMENTS}

We would like to thank Emmanuel GRESSET from Philips Semiconductors Sophia for his help to work out this project and for the fruitful discussion we had with him. This work is partially sponsored by the ADEME France and the Region PACA. 